Proc. of the 15th Int. Workshop on Slow Positron Beam Techniques and Applications, Prague, September 2-6, 2019

\title{
Open Volumes Structure and Molecular Transport in Biopolymer Nanocomposites
}

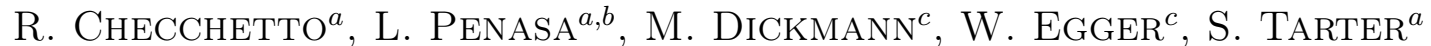 \\ AND R.S. BRUSA ${ }^{a, b, *}$ \\ ${ }^{a}$ Department of Physics, University of Trento, Via Sommarive 14, I-38123 Trento, Italy \\ ${ }^{b}$ TIFPA/INFN, University of Trento, Via Sommarive 14, I-38123 Trento, Italy \\ ${ }^{c}$ Institut für angewandte Physik und Messtechnik LRT2, Universität der Bundeswehr München, \\ Werner-Heisenberg-Weg 39, 85577 Neubiberg, Germany
}

\begin{abstract}
We discuss gas barrier mechanisms in biopolymer nanocomposite in which fillers of different natures and concentrations are added. The kinetics of gas transport is studied by gas phase permeation techniques and free volumes are analyzed by positron annihilation lifetime spectroscopy. Gas barrier properties of two biopolymer nanocomposite films are presented in relation to their free volume. The first film is poly(3-hydroxybutyrate-co-3hydroxyhexanoate) (PHBH) containing $0.25 \mathrm{wt} \%$ of graphene oxide (GO) filler nanoparticles. The second film is poly(lactic acid) (PLA) in which cellulose nanofibrils have been dispersed with content from 4.1 to 12.4 vol.\%. It is shown that in both biopolymer films the filler addition improves their gas barrier properties but with different mechanism.
\end{abstract}

DOI: 10.12693/APhysPolA.137.118

PACS/topics: 82.35.Lr, 61.25.hp, 36.10.Dr, 78.70.Bj

\section{Introduction}

Bioplastics are plastic materials that have to fulfil two conditions: to be biobased and biodegradable [1]. The present tendency is to increase the production and the use of bioplastic for reducing the dependence from the fossil resources and reducing the greenhouse gas emission. Biodegradability in soil and marine environment could also help to solve the problem of plastic waste. The consequent growth of the use of bioplastic is pushing the need of biopolymers with increasing properties for new applications.

In this paper we present a study of the gas transport mechanisms in two biopolymer membranes that have been modified with filler for increasing their barrier properties. Introduction of impermeable fillers can modify the gas transport through the membranes by different mechanisms: the fillers oblige penetrant molecule to flow through tortuous path and the permeability is reduced depending on the filler content and their aspect ratio; fillers modify the chemical-physical filler-polymeric matrix interface by rigidification of a layer or with the formation of defects [2]. Here we discuss the transport mechanisms at the light of open volume and permeation measurements. The first membrane was produced with $\mathrm{PHBH}$, a semicrystalline biodegradable polyester that can be obtained either by microorganisms in specific fermentation condition or chemical synthesis [3]. It was modified introducing $0.25 \mathrm{wt} \%$ of GO nanoparticle. The second membrane is based on PLA, an amorphous

*corresponding author; e-mail: brusa@science.unitn.it biodegradable polymer, good candidate for food packaging applications [4]. PLA nanocomposite with functionalized cellulose nanofibrils (LNC) was produced by solvent mixing in chloroform with filler content 4.1 vol.\% to 12.4 vol. $\%$.

\section{Experimental}

Details about the synthesis of PHBH, PHBH containing $0.25 \mathrm{wt} \%$ of graphene oxide (GO) filler nanoparticles (GO-PHBH), can be found in Ref. [5]. The $0.25 \mathrm{wt} \%$ was the highest GO content that was possible to add without producing very brittle PHBH film samples.

Details about synthesis of PLA and PLA with 4.1-12.4 vol.\% of LNC nanocomposite samples are reported in Ref. [6]. In samples with 5.4 vol.\% LNC, nanofibrils were observed, by SEM micrographs, to form aggregates with irregular shapes with a size of $\approx 0.8 \mu \mathrm{m}$. Increasing the content to $12.4 \mathrm{vol} . \%$, the aggregates assume an ovoid shape with size of $\approx 1.4 \mu \mathrm{m}$.

PALS measurements in PHBH $2 \mathrm{~mm}$ thick films were carried out with a lifetime fast-fast coincidence apparatus equipped with $\mathrm{BaF}_{2}$ scintillators coupled to Hamamatsu H3378 photomultiplier, total resolution 260 ps FWHM. The stop and start signals were processed with two ORTEC 583B constant fraction differential discriminators, an ORTEC 566 time to amplitude converter, and a FAST Com Tec multichannel analyzer.

PALS depth resolved measurements in neat PLA $50 \mu \mathrm{m}$ thick films, and nanocomposite PLA with LNC $4.1 \mathrm{vol} . \%$ and $9.9 \mathrm{vol} . \%$ films were done with the pulsed low-energy positron system (PLEPS) [7], at the intense positron source NEPOMUC at the research reactor FRM II [8]. Total resolution was 180 ps. PALS spectra 
were analysed with LT and PALSFIT/3 packages $[9,10]$. All spectra were well fitted with three free lifetimes, and the free hole sizes of the biopolymers were obtained by the long lifetime $\left(\tau_{3}\right)$ pertinent to Ps formation using the Tao-Eldrup model [11].

Details about permeation measurements can be found in Refs. [12] and [13]. In brief, test gases with different molecular size kept at constant pressure fill a chamber (high pressure side). The membrane sample separates the gas filled chamber from a continuously pumped chamber (low pressure side) where test gases permeate. The partial pressure of the gases permeating in the pumped chamber is measured, in transient and stationary transport conditions, with a calibrated quadrupole mass spectrometer and provides the values of the gas molecular flux as a function of time (permeation curve). In this experimental setup indeterminations can be estimated given as the average value of the background partial pressure fluctuations of the permeating gas. The gas transport through the polymeric membranes is controlled by the solution-diffusion mechanism. The analysis of the permeation curves with the Fick diffusion equation with assumption that Henry's law controls gas absorption in the membranes layers [12-14], permit to evaluate the gas diffusivity $D$ and the permeability $P=D \Pi$ where $\Pi$ is the gas solubility in the membrane layers. Measurements as a function of the temperature of the membrane, allow to construct the Arrhenius plot for diffusivity, permeability, and solubility, from which the activation energies of the three processes can be obtained.

\section{Results and discussion}

\subsection{PLA and PLA nanocomposite}

Thermal transitions investigated on PLA and PLA + LNC by differential scanning calorimetry (DSC) showed that the glass transition temperature $T_{g}$ changed only by 1 degree (from 57 to $58^{\circ} \mathrm{C}$ ) with addition of CNF. Small change is also observed for the melting temperature $T_{m}$ that changes from 165.9 to $166.7^{\circ} \mathrm{C}$.

The permeability $P$, measured at $T=310 \pm 1 \mathrm{~K}$, as a function of the LNC content is reported in the upper part of Fig. 1 for two test gases. Permeability of PLA with 5.4 vol.\% is lower than permeability of pure PLA (i.e. PLA $0 \%$ LNC in the figure), indicating a small barrier effect, then permeability increases exceeding the values of pure PLA. The diffusivity values are found to be practically constant, see lower part of Fig. 1.

In Fig. 2, we report the PALS measurements as a function of temperature for PLA and PLA with 4.1 vol.\%, 9.9 vol.\% of LNC. In Fig. 2a the long lifetimes $\tau_{3}$ and the conversion in the hole size of their values are shown, while in Fig. 2b the intensity $I_{3}$ of the $\tau_{3}$ lifetime is reported.

The hole radius values in pure PLA start from $\approx 0.285 \mathrm{~nm}$, then increases monotonically with the temperature up to a value of $\approx 0.295 \mathrm{~nm}$. The increase of hole size with temperature is distinctive of the free

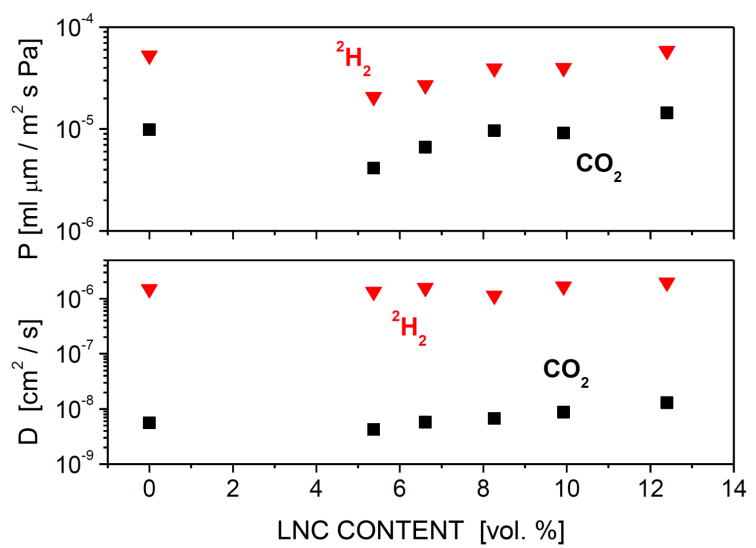

Fig. 1. Permeability (upper part) and diffusivity (lower part) values as a function of the filler vol.\% content in PLA for deuterium and carbon dioxide. Indeterminations are inside the size of the symbols.

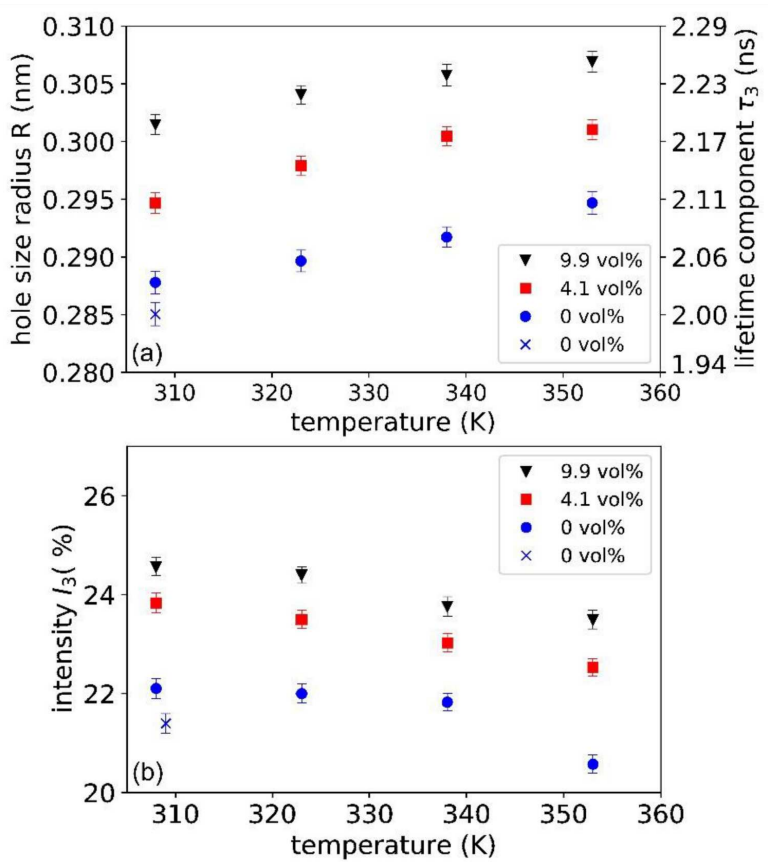

Fig. 2. Hole size radius and $\tau_{3}$ lifetime values (a) and intensity of $\tau_{3}$ lifetime (b), for PLA and PLA $+\mathrm{LCN}$ 4.1 vol.\%, PLA+LCN 9.9 vol.\%.

volumes behavior in polymeric matrix. Adding LNC, the hole size increases up to $0.3 \mathrm{~nm}$, see point at RT in Fig. 2a. In membranes with LNC addition the increase of hole size with $T$ is monotonic and similar to that in pure PLA.

Lifetime in cellulose nanofibrils film was previously measured [15]. It was found that channels of $0.15 \mathrm{~nm}$ radius are formed among nanofibrils. The corresponding Ps lifetime was of $1.4 \mathrm{~ns}$ and consequently possible Ps annihilation in LNC fillers cannot explain the increase in lifetime observed in the present PLA + LNC. 
Combining this two observations, we can conclude that the increase of lifetime due to the inclusion of LNC in PLA is likely due to formation of larger cavities at the filler-matrix interface. The size of these cavities seem not to be strongly influenced by the increase of sample temperature, because the observed variation as a function of $T$ is similar to that of pure PLA and so can more be ascribed to the increase in hole size of the PLA matrix (see Fig. 1a).

This microstructural description is also supported by the analysis of the permeation data that are well fitted by a model considering a region around the ovoid filler with permeability higher than that of PLA [6].

The decrease in lifetime intensities, see Fig. 2b, can be inferred to be related to a slight modification of the PLA structure after the measurement at the highest temperature. Lifetime measurement at RT after the measurements at increasing temperature showed that the intensity and the lifetime values were slower, see crosses in Fig. 2. The modification of the structure is supported by thermal desorption measurements that indicates that chloroform molecules start to be desorbed by PLA and $\mathrm{PLA}-\mathrm{LCN}$ composite from $\approx 340 \mathrm{~K}[16]$.

\section{2. $\mathrm{PHBH}$ and $\mathrm{PHBH}$ nanocomposite}

From DSC measurements on $\mathrm{PHBH}$ and $\mathrm{GO}-\mathrm{PHBH}$ the following data were obtained: $T_{g} \approx 0{ }^{\circ} \mathrm{C}$, melting temperature 136 and $146{ }^{\circ} \mathrm{C}$ for the amorphous and crystalline part, respectively. The $28 \%$ crystalline part of $\mathrm{PHBH}$ increased up to $60 \%$ with the addition of GO. $\mathrm{X}$-ray diffraction (XRD) measurements give a crystallite size of $30 \mathrm{~nm}$ along (020) crystallographic direction and $5 \mathrm{~nm}$ along (002) crystallographic directions.

Lifetime $\tau_{3}$, intensity of $\tau_{3}$, and hole radius in $\mathrm{PHBH}$ and $\mathrm{GO}-\mathrm{PHBH}$ are found to be equal within the errors (0.008 ps): $2.112 \mathrm{~ns}, \approx 20 \%, \approx 0.30 \mathrm{~nm}$, respectively. The inclusion of GO does not change the positron annihilation site, pointing out that positrons probe only

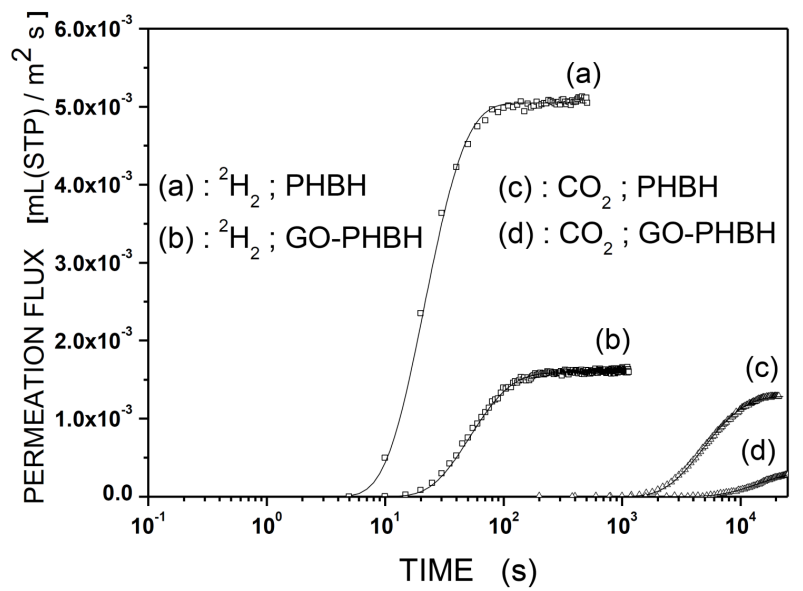

Fig. 3. Permeation curves of deuterium and carbon dioxide through $\mathrm{PHBH}$ and GO-PHBH films. Indeterminations are inside the size of the symbols. the amorphous part of PHBH. Positrons that end their thermalization path in the few nanometer size nanocrystals easily diffuse at the amorphous interface probing the amorphous matrix. Moreover, these measurements indicate that the open volume structure of PHBH amorphous part is not modified.

Permeation curves, see Fig. 3, have shown that GO inclusion reduces the permeation rates of deuterium and carbon dioxide by a factor of $\sim 3$ and $\sim 5$, respectively. Because the open volume structure is not modified the reduction in permeability is due to an increased tortuous path that gases must follow given by the presence of more dispersed impermeable crystallite and possible rigidified region around crystallite.

\section{Conclusions}

We have studied by PALS the void microstructure and by gas phase transport the permeation on two biopolymers nanocomposite. Introduction of $\mathrm{GO}$ in $\mathrm{PHBH}$ has found to be inducing an increase of the gas impermeable $\mathrm{PHBH}$ crystalline fraction that force the gas molecules to follow longer path and consequently increase the barrier property of $\mathrm{PHBH}$. While introduction of cellulose nanofibrils LNC in PLA was found to modify the structure of the PLA layers at the matrix-filler interface. After a first small decrease in permeability, the increase of LNC content worsen the barrier effect due to formation of more cavities at the filler interface that introduce easier path for permeant gases.

\section{Acknowledgments}

Part of this work is based upon experiments performed at the NEPOMUC facility at the research reactor FRM II, operated by the Heinz Maier-Leibnitz Zentrum (MLZ), Garching, Germany.

\section{References}

[1] European Bioplastics.

[2] D. Roilo, P.N. Patil, R.S. Brusa, A. Miotello, S. Aghion, R. Ferragut, R. Checchetto, Polymer 121, 17 (2017).

[3] A.K. Mohanty, M. Misra, G. Hinrichsen, Biofibers Macromol. Mater. Eng. 276, 1 (2000).

[4] C. Swaroop, M. Shukla, Int. J. Biol. Macromol. 113 729 (2018).

[5] H. Mahmood, A. Pegoretti, R.S. Brusa, R. Ceccato, L. Penasa, S. Tarter, R. Checchetto, Polym. Test. 81, 106181 (2020).

[6] D. Rigotti, R. Checchetto, S. Tarter, D. Caretti, M. Rizzuto, L. Fambri, A. Pegoretti, Express Polym. Lett. 13, 858 (2019).

[7] W. Egger, in: Proc. Int. School of Physics Enrico Fermi, Ch. CLXXIV, Physics with Many Positrons, Eds. R.S. Brusa, A. Dupasquier, A.P. Mills, NorthHolland, Amsterdam 2010, p. 419. 
[8] C. Hugenschmidt, in Ref. [7], p. 399.

[9] J. Kansy, LT Programs, LT Polymers.

[10] J.V. Olsen, P. Kirkegaard, M. Eldrup, Technical University of Denmark, PALSfit3.

[11] M. Eldrup, D. Lightbody, J.N. Sherwood, Chem. Phys. 63, 51 (1981).

[12] R. Checchetto, R.S. Brusa, Acta Phys. Pol. A 132, 1490 (2017).

[13] R. Checchetto, P. Bettotti, R.S. Brusa, G. Carotenuto, W. Egger, C. Hugenschmmidt, A. Miotello, Phys. Chem. Chem. Phys. 21, 15471 (2019).
[14] R. Checchetto, Int. J. Polym. Sci., 4903904 (2018).

[15] D. Roilo, C.A. Maestri, M. Scarpa, P. Bettotti, W. Egger, T. Koschine, R.S. Brusa, R. Checchetto, J. Phys. Chem. C 121, 15437 (2017).

[16] R. Checchetto, D. Rigotti, A. Pegoretti, A. Miotello, Pure Appl. Chem., 20181216 (2019). 